\title{
Exo-Esoteric Knowledge and the Evolution of Transmission Fidelity
}

\author{
Adrian V. Bell ${ }^{* 1}$ and Timothy M. Waring ${ }^{2}$ \\ ${ }^{1}$ Department of Anthropology, University of Utah, Salt Lake City, UT, USA \\ ${ }^{2}$ School of Economics and the Mitchell Center for Sustainability Solutions, University of Maine, \\ Orono, ME, USA
}

Keywords: evolution of teaching · cultural transmission · game theory · exoteric-esoteric knowledge $\cdot$ cultural evolution

\begin{abstract}
Teaching is a sophisticated form of cultural transmission believed to increase transmission fidelity over less formal or less intentional modes of transmission. As such, teaching may have contributed to the build-up and maintenance of cultural complexity in human societies. However, teaching is also a strategic and dynamic behavior that likely evolves in response to both the type of knowledge taught and the social context. We explore this possibility through an evolutionary analysis of teaching fidelity, or the fidelity of knowledge transmission during teaching.

For our analysis we developed two models, a two-trait model and a quantitative trait model to explore how selection on teaching fidelity varies across different types of knowledge. In our two-trait model we use exoteric and esoteric knowledge categories and specify them mathematically to ask how they may affect the evolutionarily-favored level of teaching fidelity. We show that the evolutionarily-stable transmission fidelity by teachers varies widely across knowledge types and depends on the number of students belonging to a teacher. Esoteric knowledge often converges on either very high or very low fidelity teaching practices, although what is taught often follows majority belief. Along exoteric knowledge domains, increasingly high-cost (or less beneficial) behaviors predicts teachers to impart the more benign (or more beneficial) behaviors. Further, when interactions between students are characterized by a social dilemma, increases in the fraction of the student population taught by a single teacher should increase the frequency with which teachers impart the group-beneficial behavior. Our quantitative model replicates many of the insights above, and further shows selection
\end{abstract}

\footnotetext{
${ }^{*}$ Corresponding author

Address: 270 S. 1400 E. Rm 102 Salt Lake City, UT 84112

Email: adrian.bell@anthro.utah.edu
} 
favoring for "vague" or low fidelity teaching once broadly distributed payoffs to traits become multi-modal.

Our analysis suggests future empirical work to document the contexts of knowledge exchange and teaching styles in relation to the type of knowledge transmitted. More generally, it suggests more work to understand the evolution of teaching and teaching institutions, as their dynamics and strategic variability may be more profound than previously supposed.

\section{Introduction}

Culture areas, then, are not only a product of past history: in a very real sense, they are being made now, by the efforts of different intellectuals elaborating different kinds of knowledge (Barth, 1990, p. 650).

Central to defining the role of cultural transmission in human evolution is an understanding of how societies, institutions, and individuals store and transmit disparate categories of knowledge. Recent theoretical work suggests that teaching is vital to the accumulation of knowledge that favored the evolution of complex culture, by increasing transmission fidelity (Castro and Toro, 2014; Fogarty et al., 2011), or how accurately knowledge is passed from "teacher" to "student" (Cavalli-Sforza and Feldman, 1981, p.5657). Teaching behaviors such as direct active teaching (Kline, 2014), teaching institutions such as the master-apprentice relationship (Foray, 2004; Coy, 1989), and patterns of parental pedagogy (Tehrani and Riede, 2008) may have emerged, due to their positive influence on the fidelity of cultural transmission across generations. If successful teaching increases the fidelity of knowledge transmission, it may improve the accumulation and quality of complex adaptive knowledge in a population, and may therefore have been under selection for its beneficial effects to individuals and populations (Csibra and Gergely, 2011; Dean et al., 2012; Morgan et al., 2015). However, this description does not address the empirical literature suggesting teaching strategies also evolve, influenced by the social interactions surrounding the use of specific categories of knowledge.

\section{Teaching strategies}

Evidence across disciplines suggest teaching institutions are shaped by the type of knowledge passed on and may not always lead to high transmission fidelity. In cognitive studies, recent experiments show biases in the transmission of social versus technical variation (Mesoudi, 2009). In formal Western education, researchers have gathered a considerable amount of evidence of discipline-specific epistemology, e.g. differences in research, teaching and learning practices (Hativa and Marincovich, 1995). For instance, in the "pure" disciplines (e.g. physics), faculty were more likely than those in "applied" areas (e.g. engineering) to discuss the use of conflicting evidence, counterexamples and alternative explanations (Donald, 1990, 1995). Students in applied fields more often believe that 
knowledge constitutes a collection of facts than those in pure fields (Paulsen and Wells, 1998). For example, student study strategies in medical school were more likely to rely on rote memorization than students in psychology or social history (Entwistle, 1995; Entwistle and Entwistle, 1991). Among several typical student beliefs about the learning process in early mathematics education are these: (a) mathematics problems have one and only one right answer, (b) there is only one correct way to solve any mathematics problem - usually the rule the teacher has most recently demonstrated to the class, and (c) ordinary students cannot expect to understand mathematics; they expect simply to memorize it and apply what they have learned mechanically and without understanding (Corte et al., 2002). In contrast, the student experience in "soft fields" (e.g. social science and arts/humanities) were more likely than in hard fields (e.g. engineering and business) to rely on independent reasoning, to believe that knowledge is uncertain, and that learning is not an orderly process (Hofer, 2000; Jehng et al., 1993; Paulsen and Wells, 1998). One explanation for these differences is that traditions of teaching vary by discipline. The explanation we explore here is that teaching traditions evolve in response to the type of knowledge that each purveys.

In anthropological studies, teaching and learning not only involve novices working to master sets of information, but also include rules for discussing and gaining access to information. Most generally, teaching is considered part of the socialization process by which individuals become members of a particular cultural group (Pelissier, 1991). In this view, when teachers and learners interact with each other and with other group members, they not only contribute to the distribution of knowledge, but also change its use and meaning. For example, Odden (2011) argues that since much of cultural learning in Samoa is facilitated through observation, cultural knowledge would be highly asymmetric within this hierarchical society in the absence of a "leveling" mechanism such as formal schooling.

A prominent example is Fredrik Barth's early comparative pedagogical ethnography exploring the proximate mechanisms driving the diversity of cosmological beliefs (Barth, 1990). Barth compared the teaching behaviors of religious experts in New Guinea with those of southeast Asia. Barth was concerned with both the effect of teaching styles on belief diversity and with the contextual factors that influence the success of those styles. Two idealized teaching roles emerge from Barth's comparison. The initiator, or "Conjurer," of New Guinea engages in a ritualistic exchange with novices which is designed to impress, and to convey the depth of his exclusive knowledge in a manner which elevates the initiator. These rare displays serve to mark special events, and require skill to execute. The nature of the role of the initiator, demands that he build and protect the uniqueness of his skills, because if others can successfully imitate him, he looses value in society.

While the status of the Conjurer depends on imparting a sense of connection to secret mysteries, the "Guru" of the Asian tradition, e.g. imams of Indonesia and Pakistan, depends on just the opposite - imparting a sense of wisdom conveyed. The Guru engages in 
long-term relationships with many disciples for whom he strives to deliver a sophisticated body of complex knowledge. Passages from religious texts, such as the Koran, may be repeated and interpreted as instructed the Guru. In such a milieu, individuals are forced to seek behaviors and beliefs that match with those of the Guru as well as with those of others in society at large. Thus, "Gurus" provide wisdom through the structured mastery of knowledge, while "Conjurers" impart a sense of shared secrets. Barth's description of these idealized teaching roles is useful because it helps to clarify one potential pattern of the coevolution between institutions, knowledge, and teaching fidelity.

This combined body of research suggests that teaching fidelity is influenced by patterns of social value and organization, and thus comes to reflect how knowledge is used in society. As a result, we anticipate that teaching strategies may be contingent on multiple factors, influencing the role of teaching in increasing human adaptability.

\section{The esoteric-exoteric knowledge continuum}

Formal theoretical work to date models the evolution of social learning strategies in order to pose questions about human adaptability (Boyd and Richerson, 1985; Nakahashi et al., 2012), yet no formal evolutionary theory explicitly investigates the evolution of teaching styles and fidelity. We aim to fill this gap through a mathematical caricature of the way knowledge is used in different types of social interaction, and its influence on teaching fidelity. To accomplish this develop a transparent way to follow the coevolution of knowledge and teaching strategy in different social conditions.

Of the many ways in which knowledge has been classified, one of the most fundamental is the exoteric-esoteric continuum. Exoteric and esoteric knowledge categories are used differently across disciplines, such as in the philosophy of science (e.g. Fleck, 1935), folklore (e.g. Jansen, 1959), and anthropology (e.g. Boas, 1940; Wassmann, 1991). However, the common understanding is that different types of knowledge may display a different distribution across the population. Thus, exoteric, or "external" knowledge tends to be distributed widely across a population, while esoteric, or "internal" knowledge is often shared among a specialized few. Exoteric knowledge may be often be of a more "applied" nature, while esoteric knowledge tends to be more abstract (i.e. Ingham, 1997, pp. 158159). Exoteric knowledge includes accumulated knowledge leading to the engineering of bridges, buildings, and design of political institutions, for example. Exoteric knowledge must be shared to work. In contrast, esoteric knowledge is used by a smaller number of individuals of a certain specialized group to achieve a socially desirable status (Wassmann, 1991, ch. 2). One purpose of esoteric knowledge is to gain prestige through a unique display of artistic/scholastic ability. In contrast to exoteric knowledge, the outcomes of the application of esoteric knowledge may be ambiguous, and the dimensions of meaning may not be quickly discerned. Although exoteric-esoteric continuum is useful as a rhetorical device it has never been explored in a model of knowledge evolution. How do different 


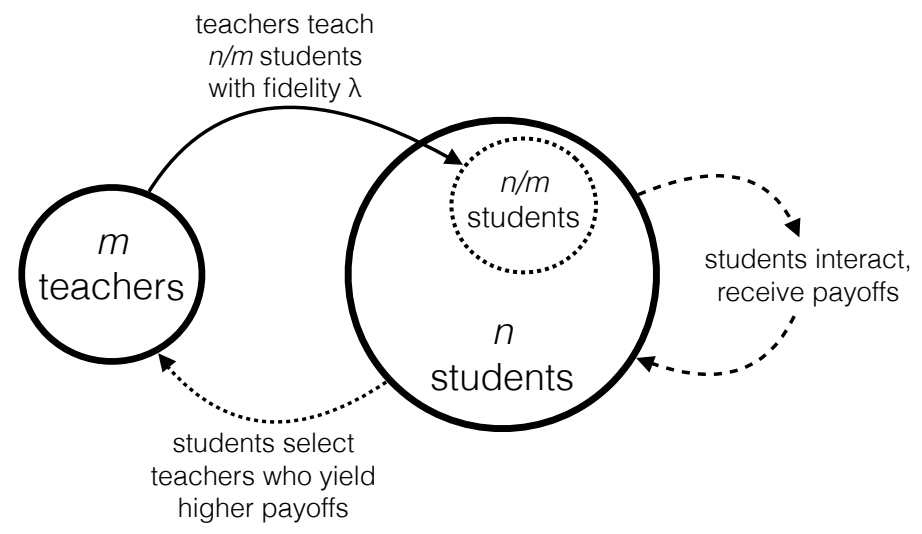

Figure 1: The social and population-level processes of the model.

types of knowledge achieve their distributions in society as teaching itself evolves?

Based on two premises, that the use of knowledge shapes its transmission and the exoteric-esoteric continuum accounts for much of knowledge variation, we develop and analyze a mathematical model to investigate the evolution of transmission fidelity among teachers. We make explicit distinct incentive structures prescribed by exoteric and esoteric knowledge and ask how it influences teaching transmission fidelity. We approach the modeling in two parts. First we consider the teaching of two alternative traits, allowing analytical tractability. Then we consider the teaching of a quantitative trait, with simulations showing the variation produced by optimal teaching strategies. Through this analysis we show that teaching fidelity may vary widely, and the diversity of beliefs also, depending on shifting incentive structures reflecting how knowledge is used. We then suggest future lines of theoretical and empirical work.

\section{Model of teaching two alternative traits}

\section{Model Specification}

The model presents a simple world in which teachers employ pedagogical strategies that influence how accurately their knowledge is transmitted to students. Students use the knowledge they have learned in their interactions with their peers in society. The results of these interactions influence student success and depend on student knowledge and the type of the interaction. The success of students is therefore related to the strategy of their teacher. Finally, we assume that through conscious selection or otherwise, new students bias their affiliation to teachers who have a more positive influence on student payoffs. Teachers benefit from a greater number of followers. As a result, a given teaching strategy and its associated transmission fidelity will be favored by selection and increase in frequency if it provides the greater relative payoff to students. Calculating the fitness 


\section{Student Peer}

\section{Focal Student}

\begin{tabular}{c|c|c|}
\multicolumn{1}{c}{} & \multicolumn{1}{c}{ Belief $A$} & Belief $B$ \\
\cline { 2 - 3 } Belief $A$ & $a$ & $b$ \\
\cline { 2 - 3 } Belief $B$ & $c$ & $d$ \\
\cline { 2 - 3 } & &
\end{tabular}

Figure 2: The interaction matrix gives shows the payoffs to a focal student in an interaction with a peer. Payoffs $a, b, c$, or $d$ depend on the beliefs, $A$ or $B$, of both students. Different specifications of these payoffs change the nature of student interactions, influencing the choice of teachers and the equilibrium teaching strategy.

of a level of teaching fidelity this way follows widely adopted assumptions behind the evolution of social learning biases - that selection shapes human decision-making to chose from whom to learn from (Boyd and Richerson, 1985; Chudek et al., 2012; Efferson et al., 2008; Henrich and Gil-White, 2001; McElreath et al., 2008; Nakahashi et al., 2012).

(Make clear: in the model teacher and student numbers are held constant.)

Every student in a population of $n$ students learns one of two types of beliefs, A or B from a smaller population of $m$ teachers. Teaching strategies are characterized by transmission fidelity, $\lambda$, which varies between 0 and 1 such that when $\lambda=1$ belief $\mathrm{A}$ is taught with perfect fidelity, at $\lambda=0$ belief $\mathrm{B}$ is taught with perfect fidelity, and when $\lambda=0.5$ the two beliefs are taught at random. This way, students gain belief $\mathrm{A}$ from the a teacher with probability $\lambda$, and belief B with probability $1-\lambda$. Students use this acquired knowledge to interact in pairs, and students will gain benefits or suffer costs as a result of that interaction. The population structure and transmission patterns are summarized in Figure 1, and the outcomes of dyadic student interactions is given by the payoff matrix in Figure 2.

Let us assume for a moment that all teachers teach with fidelity level $\lambda$. We can now ask how fidelity $\lambda$ responds to the outcomes of student interactions and student selection of teachers. To answer this question we introduce a rare (mutant) teaching strategy $\lambda^{\prime}$. If $\lambda$ is the common teaching strategy, then the rare teacher with strategy $\lambda^{\prime}$, who teaches $m$ students, will have a fitness defined as

$$
\begin{aligned}
W\left(\lambda^{\prime}\right) & =w_{0}+a\left((1-k) k \lambda^{\prime} \lambda+k^{2} \lambda^{\prime 2}\right) \\
& +b\left((1-k) k \lambda^{\prime}(1-\lambda)+k^{2} \lambda^{\prime}\left(1-\lambda^{\prime}\right)\right) \\
& +c\left((1-k) k\left(1-\lambda^{\prime}\right) \lambda+k^{2}\left(1-\lambda^{\prime}\right) \lambda^{\prime}\right) \\
& +d\left((1-k) k\left(1-\lambda^{\prime}\right)(1-\lambda)+k^{2}\left(1-\lambda^{\prime}\right)^{2}\right)
\end{aligned}
$$

where $k=m / n$ and $w_{0}$ is baseline fitness for teachers. An invasion and stability analysis reveals the conditions under which a given level of fidelity is stable (resists invasion by another strategy) in the population, given these interactions (Maynard Smith, 1982; 
McElreath and Boyd, 2007). It follows the evolutionarily stable level of teaching fidelity $\left(\lambda_{E S S}\right)$ is,

$$
\lambda_{E S S}= \begin{cases}\frac{(k c+b)-d(1+k)}{((c+b)-(a+d))(1+k)} & \text { if } a+d<b+c \\ \{0,1\} & \text { otherwise. }\end{cases}
$$

We use this general result and ask what teaching strategy is favored as we change the nature of student-student interactions. To do this we specify different values of $a, b, c$, and $d$ shown in Figure 2 that characterize the social interactions reflected in the use of esoteric and exoteric knowledge. This approach reflects the presumption that knowledge evaluation can be represented as a social process where, for example, 'useful' knowledge can either be widely shared among individuals in a population, or only shared with a specialized few (Barnes et al., 1996). We employ this general model to explore two unique and interacting dimensions relevant to knowledge transmission: external validity and social coordination.

Exoteric knowledge is defined as having some external validity, and can therefore be independently ascertained, such that one belief may be more correct or beneficial than another belief in objective terms. For example, the correct way to prepare a poisonous fish for eating or the location of a water source are knowledge types that likely become exoteric. Thus, in a domain of exoteric knowledge, one belief may yield an objective higher payoff to its holder than another. By contrast, we define esoteric knowledge as knowledge that is not externally or objectively verifiable, but may still accumulates benefits through social interactions. For example, local religious knowledge or a proper greeting are types of esoteric knowledge. We use this basic contrast to construct alternative types of student interaction in each knowledge domain. Additionally, we vary the relationship between the group-beneficial behavior and individually-beneficial behavior. A coordination game results when individual best-choices match group best-choices, while a social dilemma occurs when the two conflict. The two dimensions of external validity and social coordination yield three fundamental cases which we use to explore the evolution of teaching strategies (see Table 1).

Exoteric knowledge conditions. To model exoteric knowledge, we develop two student interactions, Eso-Exo Coordination and Exoteric Dilemma, in which optimal beliefs are objectively ascertainable to adjustable levels. In Eso-Exo Coordination we specify payoffs so that when both students hold belief $\mathrm{A}$, the highest benefits accrue to both individuals as well as the group as parameter $\delta_{c}$ increases (see Table 1). Exoteric dilemma is a cooperative social dilemma in that sharing the same behavior $A$ gives the largest group benefit (i.e. the cooperative option), but the higher individual benefit comes from holding behavior $B$ when one's peers hold otherwise. The payoff parameters are given in Table 1 . 


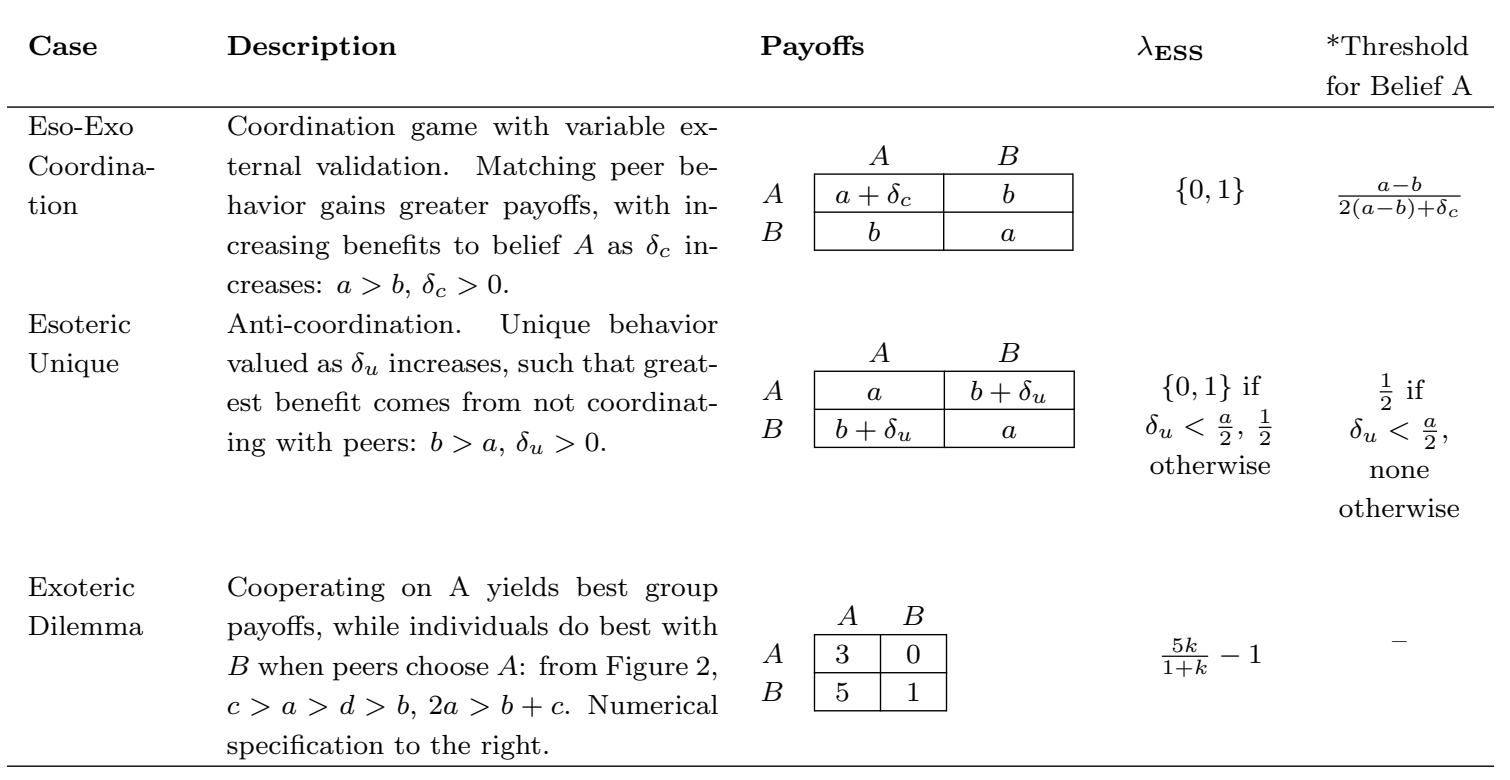

Table 1: Mathematical specification of esoteric and exoteric knowledge categories with their respective evolutionary-favored teaching fidelity $\left(\lambda_{\mathrm{ESS}}\right)$. ${ }^{*}$ When high teaching fidelity is favored for either belief A or $\mathrm{B}\left(\lambda_{\mathrm{ESS}}=\{0,1\}\right)$, the far right column specifies the minimum frequency of belief $\mathrm{A}$ among students required for belief $\mathrm{A}$ to be taught exclusively. See further discussion of the threshold for belief A, in the text.

Esoteric knowledge conditions. Esoteric specifications require knowledge that is not externally or objectively verifiable but in which payoffs are determined solely by the factors of social interaction. The Eso-Exo Coordination specification is adjustable to represent an esoteric condition, where having the same belief as your peer provides the highest payoff (as $\delta_{c}$ goes to zero). This constitutes a pure coordination game, such as might be found in adopting common practices for social exchange. In contrast, Esoteric Unique is a specification where having the same belief is very costly. Higher payoffs go to individuals with different beliefs from those with whom they interact, and even more so as the 'uniqueness' parameter $\delta_{u}$ increases. These representations approximate the social conditions that Barth described amongst Melanesian initiators or 'conjurers.' These specifications also satisfy the esoteric factor of either needing to match knowledge with others, or to show individualism through disparate beliefs (see Table 1 for payoff structure).

\section{Model Results}

The evolutionarily stable level of teaching fidelity $\left(\lambda_{\mathrm{ESS}}\right)$ is highly dependent on payoff parameters and sometimes the number of students taught by a teacher. Teaching fidelity reflects the student response to the type of social interaction and the type of knowledge at play (Table 1). When the social interaction is one of coordination or anti-coordination, Eso-Exo Coordination and Esoteric Unique, the ESS teaching strategy is to teach at the 

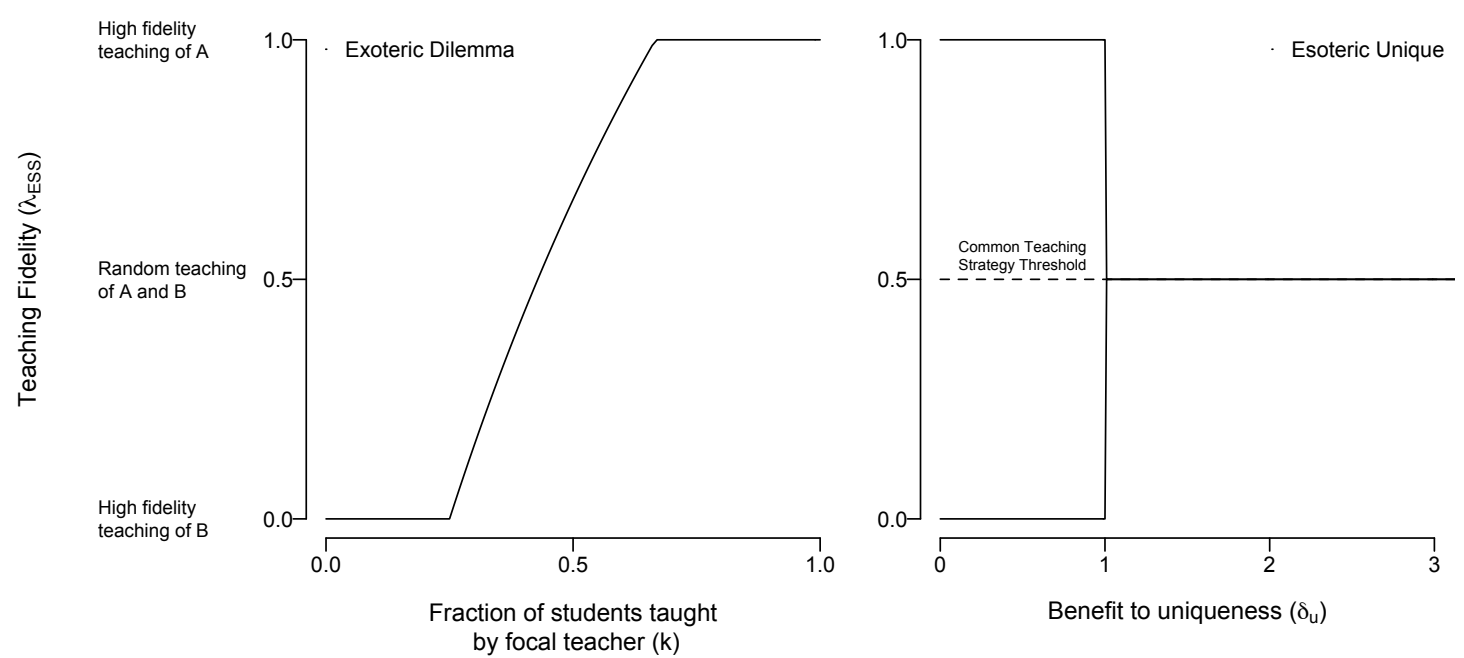

Figure 3: Plot of ESS levels of teaching fidelity for Exoteric Dilemma and Esoteric Unique knowledge categories, using results from Table 1. Students gain belief A from a teacher with probability $\lambda$, and belief $\mathrm{B}$ with probability $1-\lambda$. The right panel shows a region where $\lambda_{E S S}$ is $\{0,1\}$, and an intermediate value $\lambda_{E S S}=1 / 2$, with the former characterized by a threshold (dashed curve). When the frequency of peers holding belief A or B is above or below the threshold, belief A or B, respectively, is favored to be taught with the highest fidelity.

extremes for coordination, either belief $\mathrm{A}$ or $\mathrm{B}\left(\lambda_{\mathrm{ESS}}=\{0,1\}\right)$, or teach beliefs $\mathrm{A}$ and $\mathrm{B}$ randomly (Figure 3). Thresholds based on coordination payoffs specify when a frequency of belief among students dictates which behavior teachers pass on (Figure 4). In the esoteric knowledge coordination scenario (Eso-Exo Coordination with $\delta_{c}=0$ ), there is no objective external difference between the two beliefs, the threshold is thus $q_{T}=1 / 2$, and which belief is favored to teach is that which is more common among peers. By contrast, when coordination is involved in a domain where knowledge is externally verifiable (EsoExo Coordination with $\delta_{c}$ at larger values), the more beneficial belief $\mathrm{A}$ is favored over belief B, with the threshold,

$$
q_{T}=\frac{a-b}{2(a-b)+\delta_{c}}
$$

If the frequency of students with belief $\mathrm{A}$ is above threshold (3) belief $\mathrm{A}$ is favored, $\mathrm{B}$ otherwise. Which belief becomes fixed in the population depends on the history of the population, such as founder effects or cultural drift. Clearly belief A, the externallyvalidated belief when $\delta_{c}>0$, has a larger basin of attraction (Figure 4). However, the ratio of students to teachers may also determine the teaching of behavior $A$ or $B$. This is shown in the Exoteric Dilemma condition. Here, as the fraction of students taught by a teacher increases $(k \rightarrow 1)$, teaching the group-beneficial behavior (belief A) becomes more favored 


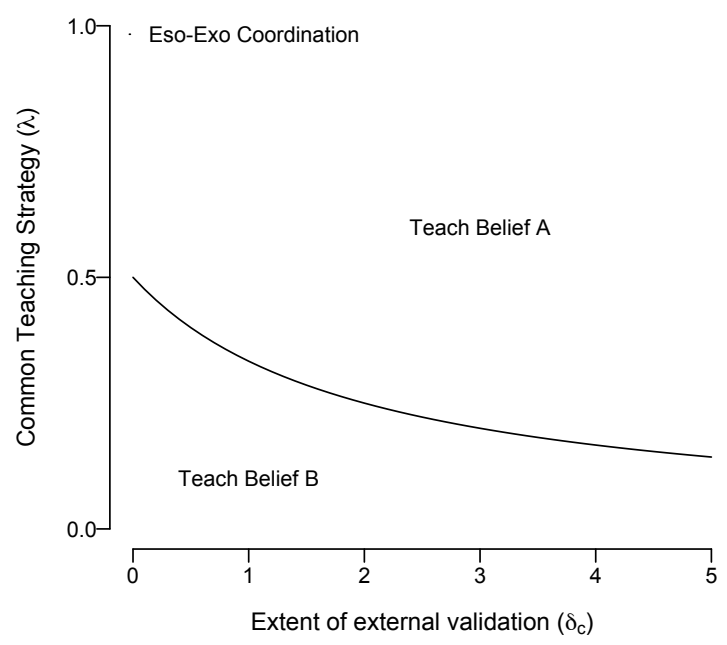

Figure 4: Favored teaching strategy under Eso-Exo Coordination using results from Table 1, depending on the strength of external validation $\left(\delta_{c}\right)$ and the common teaching strategy $(\lambda)$. The curve represents a threshold, where above the curve high-fidelity teaching of belief A is favored, and below the curve high-fidelity teaching of belief $\mathrm{B}$ is favored.

(Figure 3). This is because students with belief A interact more frequently with students of the same type, and receive the higher benefit. Thus we see that when coordination is involved with an externally validated belief, the spread of exoteric knowledge is enhanced as the ration of students to each teacher increases.

In sum, these results describe at least two important ways knowledge categories shape teaching strategies. First, if there is not a genuine external benefit to one or the other belief, it only matters what everyone else is doing to know what to teach. The exception is that increasingly high-cost behaviors should induce teachers to impart the more benign (or externally-validated) behaviors. However, there is a large region of the parameter space for teachers to impart social norms rather than objective truths (Figure 4). Second, as a larger fraction of the student population is taught by a single teacher (defined outside the model here), that teacher should teach the group-optimal behavior.

\section{Model of teaching a quantitative trait}

\section{Model Specification}

We now consider the case where teachers facilitate learning a quantitative trait. In this way we better assess what levels of teaching fidelity are favored when imparting traits with a continuous range of weak to strong social or exogenous benefits. Following Boyd 
\& Richerson $(1985,:$ 100), the student payoff to a quantitative trait may be expressed as,

$$
\pi(y)=a \exp ^{-\frac{(y-\bar{z})^{2}}{2 S_{c}}}+b \exp ^{-\frac{(y-u)^{2}}{2 S_{e}}}
$$

where $y$ is the trait gained by a student through teaching, $\bar{z}$ the average trait in a population, and $u$ is the trait value maximizing an exogenously-defined benefits. Parameters $1 / S_{e}$ and $1 / S_{c}$ determine the spread of benefits around the social and exogenous benefits, with larger values indicating a large "tolerance" by which individuals may still gain significant benefits if they deviate from the optimum $\bar{z}$ and $u$ (Boyd and Richerson, 1985, : 103). Parameters $a$ and $b$ scale the absolute fitness benefits. The trait $y$ is imparted by a focal teacher as a random normal variate with mean $\bar{y}$ and standard deviation $S_{y}$. Selection on teaching strategies will act on values of $\bar{y}$, or what is taught on average, and $S_{y}$, the standard deviation in what is taught on average, or inverse of teaching fidelity. Following assumptions in the first model that teacher success is proportional to student payoffs, the fitness of a teaching strategy becomes,

$$
W\left(\bar{y}, S_{y}\right)=\frac{1}{\sqrt{2 \pi S_{y}}} \int_{-\infty}^{\infty} \pi(y) \exp ^{-\frac{(y-\bar{y})^{2}}{2 S_{y}^{2}}} d y
$$

which is the average fitness of a teacher with strategy $\bar{y}$ and $S_{y}$. Essentially, this specification models the benefits of a teaching distribution over two exogenously determined distributions. After integrating (5), our fitness expression becomes,

$$
\begin{aligned}
W\left(\bar{y}, S_{y}\right)= & a \sqrt{S_{y} \frac{S_{c}}{S_{c}+S_{y}^{2}}} \exp ^{\frac{\bar{y}^{2}}{2 S_{y}^{2}}\left(\frac{S_{c}}{S_{c}+S_{y}^{2}}-1\right)+\frac{S_{y}^{2} \bar{z}(2 \bar{y}-\bar{z})}{2 S_{y}^{2}\left(S_{c}+S_{y}^{2}\right)}} \\
& +b \sqrt{S_{y} \frac{S_{e}}{S_{e}+S_{y}^{2}}} \exp ^{\frac{\bar{y}^{2}}{2 S_{y}^{2}}\left(\frac{S_{e}}{S_{e}+S_{y}^{2}}-1\right)+\frac{S_{y}^{2} u(2 \bar{y}-u)}{2 S_{y}^{2}\left(S_{e}+S_{y}^{2}\right)}}
\end{aligned}
$$

We analyze this fitness function below.

\section{Model Results}

We explored values of $\bar{y}$ and $S_{y}$ to understand how they affect the fitness of teachers imparting a quantitative trait $y \sim N\left(\bar{y}, S_{y}\right)$, assuming contrasting values of $\bar{z}, u, S_{c}$, and $S_{e}$. When the exogenous and social payoffs are the same $(\bar{z}=u)$, then selection favors $\bar{y}$ to center on the values that maximizes payoffs. This is expected. However, due to the distributional spread around exogenous and social payoffs $\left(S_{c}\right.$ and $\left.S_{e}\right)$, the transmission variance is still nonzero, meaning some variation in what is taught is favored, .

When the exogenous and social optimum are not the same we found two major salient themes. First, when selection for social coordination and environmental adaptation is strong and equal $\left(S_{c}=S_{e}\right)$, then selection favors teaching on average either the social or environmentally behavior that maximizes payoffs, or both. Figure 5 plots teacher fitness as a function of trait value $(\bar{y})$ and teaching fidelity $\left(S_{y}\right)$. With the left panel specified with 

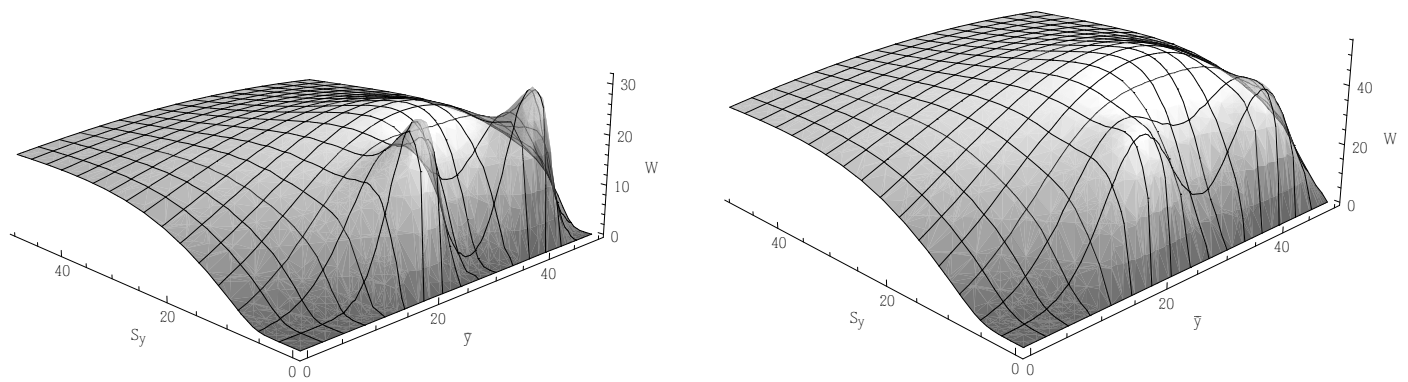

Figure 5: Fitness landscape as it varies with what is taught on average $(\bar{y})$ and its transmission fidelity $\left(S_{y}\right)$. Plot of result (6) with both panels having parameter values $\bar{z}=40, u=20$, and $a=b=30$. Selection is relatively stronger for the left panel with $S_{c}=5, S_{e}=5$, while weaker selection for the right panel with $S_{c}=20, S_{e}=20$.

peaked distributions for the social and environmental traits, we consequently see two tall fitness peaks marking a teacher's optimal strategy. Here, selection favors either the social or exogenous benefits peak, noting not with perfect fidelity. There is another peak that suggests teaching a trait intermediate between the two social and environmental peaks, yet it is a local optimum.

The second major them is the following. As the spread around exogenous and social payoffs increases, then selection favors teaching an intermediate trait with much greater variability, or much less teaching fidelity. The right panel of Figure 5 shows an increase in height of the "intermediate" peak relative to the left panel, becoming the global optimum. This peak's location is further along the $S_{y}$ axis, indicating selection for lower teaching fidelity. This indicates that as the spread of payoffs for two contrasting forces increases, then teaching fidelity declines considerably.

We see the effects of payoff spread more clearly in Figure 6. In the left panel, as the spread of payoffs increases for both exogenous and social benefits, selection favors teaching an intermediate trait on average (closer to 30) between the two exogenously determined traits. Not surprisingly selection also favors the traits with less payoff spread represented close to the vertical and horizontal axes. However, as in the left panel of Figure 5, values closer to the origin selects for teaching either (or both) traits.

Finally, there is a large region in panel 2 of Figure 6 where transmission fidelity is low. The standard deviation of what is taught $\left(S_{y}\right)$ climbs quickly and asymptotes as the spread of payoffs increases. High fidelity transmission requires a fairly restricted payoff spread to both the exogenous and social benefit curves. 

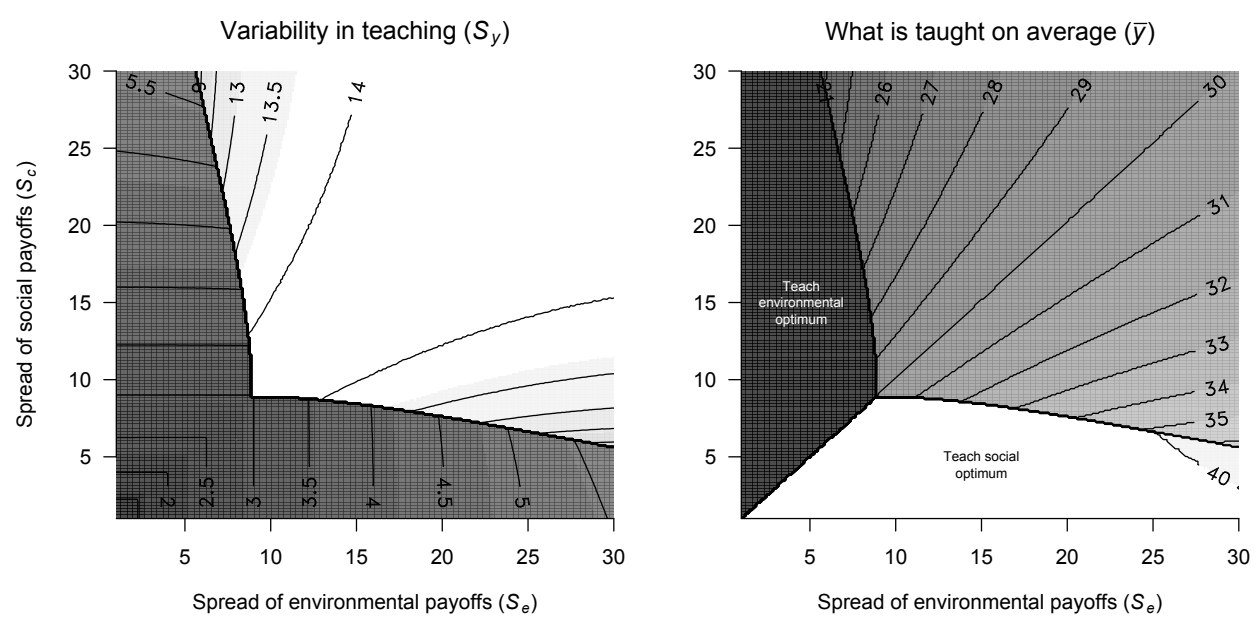

Figure 6: What natural selection would favor being taught on average $(\bar{y}$, left panel) and its fidelity ( $S_{y}$, right panel) as a function of the spread of social and environmentally-determined payoffs. Contours describe the maximum fitness value of (6) with respect to $\bar{y}$ and $S_{y}$. Both panels having parameter values $\bar{z}=40, u=20$, and $a=b=30$.

\section{Discussion}

Evidence from a variety of social science disciplines describe high variability in teaching and learning styles by knowledge category. Transmission fidelity, in particular, is a salient characteristic of teaching because it greatly influences human adaptability, but is itself determined by the types of knowledge involved. Our model and analysis of the evolution of teaching fidelity verifies these observations by showing how teaching fidelity evolves according to different categories of knowledge, their verifiability and the social interactions through which they influence outcomes. Our model does not support the notion that all teaching will lead to high fidelity transmission and the accumulation of complex culture, but rather that what is taught and with what accuracy can vary widely (Figure 3).

In the esoteric or exoteric knowledge domains of our two-trait model, when coordination is relevant, teaching will tend to converge on a single belief determined by the beliefs of others. For instance, in many cases, believing what your peers believe serves you best. Our analysis suggests that in such cases, cultural selection would favor teaching the majority belief. The reverse also holds. If uniqueness determines payoffs in esoteric domains, selection would favor teaching a rare belief, accomplished through the random imparting of alternative beliefs. This is "vague" teaching. In exoteric domains, when there is an optimal belief, teachers come to teach that belief with a fidelity proportional to the fraction of students belonging to his/her "school of thought" and in proportion to the payoffs to coordinating on that belief. Thus one key finding of our model is that the demographic conditions of knowledge transfer such as student-teacher ratios, have a 
very powerful influence on teaching fidelity. This finding is in concordance with previous research discussing the rise of maladaptive culture (Richerson and Boyd, 2005, ch.5), population history, frequency-dependent dynamics, and social learning biases that likely guide the trajectory of cultural knowledge (McElreath and Boyd, 2007, pgs. 206-2015).

Our quantitative trait model affirms many of the insights of the two-trait model. Greater validation of a trait or trait distribution favors it being taught with higher fidelity. However, vague or low-fidelity teaching of "middle-ground" traits arises quickly as competing "schools of thought", environmental and/or social forces allow for a larger range of traits to garner benefits. While we did not look at more than two quantitative forces, we envision these basic insights will hold for a larger collection of forces that shape the fitness landscape of what a teacher imparts with what level of variability.

Our analysis suggests vague teaching even within quantitative/scientific domains where sociological forces are important. This finding is consistent with the sociological concept norms of interpretation that we see in the validation of highly technical knowledge, such as in the debate over determination of the charge of an electron (the Millikan-Ehrenhaft controversy discussed in Barnes et al., 1996, pgs. 18-45). This work shows convincingly that the acceptance of knowledge and inferences is ultimately determined by others who allow such to pass review.

Selection on transmission fidelity is likely to vary across different modes of teaching. Kline (2014) provides a typology, where teaching can occur through: a) social tolerance, b) opportunity provisioning, c) stimulus or local enhancement, d) evaluative feedback, and d) direct active teaching. While social traditions regarding knowledge validation are likely active across all these modes, evaluative feedback and direct active teaching are more amenable to teachers directing knowledge transmission. However, more work is needed to understand the perhaps complex relationship between knowledge categories, transmission fidelity, and modes of teaching.

While our object here is the scrutinize the relationship between teaching and transmission fidelity, we do not dispute the role teaching likely played in increasing human adaptability early in human evolution to the present, as experimental evidence suggests (Morgan et al., 2015). Figures 3 and 4 shows regions where exoteric beliefs with high payoffs are predicted to be taught with high fidelity regardless of student-teacher ratios $(k)$ and the common teaching behavior $(\lambda)$. High fidelity teaching is expected as benefits to a behavior are perceived to be greater. However, our analysis also suggests empiricists might do well to consider how teaching styles themselves evolve as a focal point of study. This analysis highlights the empirical tradition of anthropologists of knowledge who: (a) document the contexts of knowledge exchange among individuals, and (b) document the benefits and costs of teaching styles given the use of the knowledge transmitted and relevant factors of social organization (e.g. Barth, 2002).

More research is needed to better integrate mathematical theory with the empirical corpus on the coevolution of teaching strategies and knowledge in various domains. Our 
model is not definitive with respect to which dyadic interactions best approximate individual interactions of those using exoteric versus esoteric knowledge, and many other formulations may be usefully explored, and may better match certain empirical conditions. That such a mapping has not been even attempted reveals how little we know about the evolution of teaching in human societies.

\section{Conclusion}

There is a large gap in evolutionary understanding of how teaching facilitates the the transmission of culture and influences cultural complexity. Our evolutionary analysis of teaching fidelity aims to fill lacuna in our conceptual understanding of the role teaching may play. However, other knowledge categories or model specifications may also provide further insight into how knowledge is validated, transmitted, and influences the cultural fitness of teachers and students. With further theoretical development and the incorporation of the empirical data on teaching and learning strategies of disparate types of knowledge, we suspect a greater understanding of the role teaching styles play in cultural evolution.

\section{Acknowledgements}

We thank Doug Jones and Polly Weissner for comments on earlier drafts of this paper.

\section{References}

Barnes, B., Bloor, D., and Henry, J. (1996). Scientific Knowledge: A Sociological Analysis. The University of Chicago Press, Chicago.

Barth, F. (1990). The Guru and the Conjurer: Transactions in knowledge and the shaping of culture in Southeast Asia and Melanesia. Man, 25(4):640-653.

Barth, F. (2002). An Anthropology of Knowledge. Current Anthropology, 43(1):1-18.

Boas, F. (1940). The Ethnological Significance of Esoteric Doctrines. In Race, Language and Culture. The University of Chicago Press, Chicago.

Boyd, R. and Richerson, P. J. (1985). Culture and the Evolutionary Process. The University of Chicago Press, London.

Castro, L. and Toro, M (2014). Cumulative cultural evolution: The role of teaching. Journal of Theoretical Biology, 347:74-83.

Cavalli-Sforza, L. L. and Feldman, M. W. (1981). Cultural transmission and evolution : a quantitative approach. Monographs in population biology : 16. Princeton University Press, Princeton, N.J. 
Chudek, M., Heller, S., Birch, S., and Henrich, J. (2012). Prestige-biased cultural learning: bystander's differential attention to potential models influences children's learning. Evolution and Human Behavior, 33(1):46-56.

Corte, E. D., Eynde, P. O., and Verschaffel, L. (2002). "Knowing What to Believe": The Relevance of Students' Mathematics Beliefs for Mathematics Education, Chapter 15. Lawrence Erlbaum Associates, Inc., Mahwah, New Jersey.

Coy, M. W. (1989). Apprenticeship: From Theory to Method and Back Again. SUNY Series in the Anthropology of Work. State University of New York Press, Albany, N.Y.

Scibra, G. and Gergely, G (2011). Natural pedagogy as evolutionary adaptation Philosophical Transactions of the Royal Society B: Biological Sciences, 366(1567):1149-1157.

Dean, L. G., Kendal, R. L., Schapiro, S. J., Thierry, B., and Laland, K. N. (2012). Identification of the Social and Cognitive Processes Underlying Human Cumulative Culture. Science, 335(6072):1114-1118.

Donald, J. G. (1990). University professors' views of knowledge and validation processes. Journal of Educational Psychology, 82(2):242-249.

Donald, J. G. (1995). Disciplinary Differences in Knowledge Validation. Jossey-Bass Inc., San Francisco.

Efferson, C., Lalive, R., Richerson, P. J., McElreath, R., and Lubell, M. (2008). Conformists and mavericks: the empirics of frequency-dependent cultural transmission. Evolution and Human Behavior, 29(1):56-64.

Entwistle, N. (1995). Frameworks for understanding, as experienced in essay writing and in preparing for examinations. Educational Psychologist, 30:47-54.

Entwistle, N. J. and Entwistle, A. (1991). Contrasting forms of understanding for degree examinations: the student experience and its implications. Higher Education, 22(3):205227.

Fleck, L. (1935). Entstehung und Entwicklung einer wissenschaftlichen Tatsache. Benno Schwabe \& Co., Basel, Switzerland.

Fogarty, L., Strimling, P., and Laland, K. N. The Evolution of Teaching. Evolution, 65(10):2760-2770.

Foray, D. (2004). Economics of knowledge. Massachusetts Institute of Technology.

Kline, M. How to learn about teaching: An evolutionary framework for the study of teaching behavior in humans and other animals. Behavioral and Brain Sciences, forthcoming. 
Hativa, N. and Marincovich, M. (1995). Disciplinary Differences in Teaching and Learning. New Directions For Teaching and Learning. Jossey-Bass Publishers, San Francisco.

Henrich, J. and Gil-White, F. J. (2001). The evolution of prestige - freely conferred deference as a mechanism for enhancing the benefits of cultural transmission. Evolution and Human Behavior, 22(3):165-196.

Hofer, B. K. (2000). Dimensionality and disciplinary differences in personal epistemology. Contemporary Educational Psychology, 25:378-405.

Ingham, A. G. (1997). Toward a Department of Physical Cultural Studies and an End to Tribal Warfare. State University of New York Press, Albany, N.Y.

Jansen, W. H. (1959). The esoteric-exoteric factor in folklore. Fabula, 2(2):205-2011.

Jehng, J.-C. J., Johnson, S. D., and Anderson, R. C. (1993). Schooling and students' epistemological beliefs about learning. Contemporary Educational Psychology, 18(1):2335 .

Kline, M. A. (2014). How to learn about teaching: An evolutionary framework for the study of teaching behavior in humans and other animals. Behavioral and Brain Sciences, (Forthcoming):1-70.

Maynard Smith, John (1982). Evolution and the Theory of Games. Cambridge University Press, Cambridge.

McElreath, R., Bell, A., Efferson, C., Lubell, M., Richerson, P., and Waring, T. (2008). Beyong existence and aiming outside the laboratory: Estimating frequency-dependent and payoff-biased social learning strategies. Philosophical Transactions of the Royal Society B, 363:3515-3528.

McElreath, R. and Boyd, R. (2007). Mathematical models of social evolution: A guide for the perplexed. University of Chicago Press, Chicago.

Mesoudi, A. (2009). How cultural evolutionary theory can inform social psychology and vice versa. Psychological Review, 116(4):929-952.

Morgan, T. J. H., Uomini, N. T., Rendell, L. E., Chouinard-Thuly, L., Street, S. E., Lewis, H. M., Cross, C. P., Evans, C., Kearney, R., de la Torre, I., Whiten, A., Laland, K. N. (2015). Experimental evidence for the co-evolution of hominin tool-making teaching and language. Nature Communications, 6 .

Nakahashi, W., Wakano, J. Y., and Henrich, J. (2012). Adaptive social learning strategies in temporally and spatially varying environments: How temporal vs. spatial variation, 
number of cultural traits, and costs of learning influence the evolution of conformistbiased transmission, payoff-biased transmission, and individual learning. Human Nature, $23(4): 386-418$.

Odden, H. L. (2011) The Impact of Primary Schools on the Differential Distribution of Samoan Adolescents? Competence with Honorific Language. Current Anthropology, 52(4):597-606.

Paulsen, M. B. and Wells, C. T. (1998). Domain differences in the epistemological beliefs of college students. Research in Higher Education, 39(4).

Pelissier, C. (1991). The Anthropology of Teaching and Learning. Annual Review of Anthropology, 20:75-95.

Richerson, P. J. and Boyd, R. (2005). Not by genes alone: how culture transformed human evolution. University of Chicago Press, Chicago.

Tehrani, J. J. and Riede, F. (2008). Towards an archaeology of pedagogy: learning, teaching and the generation of material culture traditions. World Archaeology, 40(3):316-331.

Wassmann, J. (1991). The Song to the Flying Fox: the Public and Esoteric Knowledge of the Important Men of Kandingei about Totemic Songs, Names and Knotted Cords (Middle Sepik, Papua New Guinea). Apwitihire: Studies in Papua New Guinea Musics. National Research Institute, Cultural Studies Division, Boroko, Port Moresby. 